D.T. Wickramasinghe, L. Ferrario, and G.V. Bicknell, eds.

\title{
Numerical Simulations of MHD Winds from Accretion Disks
}

\author{
James M. Stone \\ Department of Astronomy, University of Maryland, College Park, MD \\ 20742-2421 USA
}

\begin{abstract}
The contributions that time-dependent multidimensional numerical simulations have made to our understanding of MHD winds from accretion disks are reviewed. Current simulations can be divided into four categories: (1) axisymmetric global models, (2) axisymmetric simulations of the wind only, (3) three-dimensional local models, and (4) threedimensional global models. Results from each category are discussed. Current results indicate that weakly magnetized disks are turbulent, and that this turbulence is responsible for dynamo action which amplifies the disk field. These effects may have important consequences for the production of MHD winds. We discuss the feasibility of fully three-dimensional global models that can capture these effects in the future.
\end{abstract}

\section{Introduction}

Observations have established a clear connection between jets and accretion disks in a very wide variety of astrophysical systems, from protostellar outflows to active galactic nuclei. Indeed, evidence is mounting that all jets are produced by disks. As an example of the connection between the two, recent Hubble Space Telescope images of the protostellar jet HH110 (Stapelfeldt, these proceedings) shows an edge-on flared disk seen in absorption against the bright, optical jet. With images such as these, we no longer need cartoon pictures of the jet-disk connection, as we now have direct observation.

One of the outstanding theoretical questions to address in studies of jets from disks is: what force drives the outflow? Several possibilities immediately come to mind: (1) radiation forces, and (2) magnetic forces.

Radiation forces are thought to play a role in driving outflows in high luminosity accretion sources, such as AGN, compact objects, and perhaps massive protostars. Outflow produced by continuum opacity (i.e. super Eddington accretion) has been studied by Icke (1980), and Eggum, Coroniti, \& Katz (1988). More recently, studies of the structure of disk winds produced by radiation pressure on UV resonance lines have been undertaken. This mechanism is known to produce powerful outflows in hot stars, and may have important application in hot accretion disks as well. At this conference, a number of studies of the two-dimensional (2D) and time-dependent nature of line-driven disk winds were presented (i.e. the poster presentations of Kallman \& Pereyra, and Proga, Drew \& Stone, and the invited review of Drew). 
Magnetic forces have long been suspected as producing outflows from disks by either of two effects. The first, and most straightforward, is by strong gradients in magnetic pressure (e.g. Fukue 1990). Shear in the disk will lead preferentially to toroidal fields, thus most models rely on gradients of the toroidal magnetic pressure. On the other hand, Blandford \& Payne (1982, hereafter BP) recognized that poloidal fields are very effective at driving outflows through a "magneto-centrifugal effect". If the disk plasma is frozen-in to magnetic field lines anchored to the disk and forced to corotate, one can show that if the field is inclined to the vertical by an angle of greater than $30^{\circ}$, the plasma will be flung out through centripetal forces. BP presented a self-similar model for the steady state structure of a disk wind which might result from such an effect. Since then, these models have been extended and improved by a large number of workers to include the internal structure of the disk (Wardle \& Königl 1993), to study electrodynamic regime (Lovelace, Wang, \& Sulkanen 1987), and to study the asymptotic structure (Heyvaerts \& Norman 1989; Pelletier \& Pudritz 1989). These steady state models have discovered much about the magneto-centrifugal effect, and the collimation of outflows. That steady-state wind solutions to the equations of MHD exist at all is both important and interesting; but it does raise questions such as how do real disks relax to these solutions, and are they stable. Königl provides a review of steady-state models of MHD disk winds in these proceedings.

Given the success of steady state models, what role can numerical simulations play in studying disk winds? Numerical solutions to the equations of MHD allow some of the restrictive mathematical assumptions required for steady state models (such as self-similarity) to be relaxed. Moreover, numerical methods allow the study of time-dependent and unsteady phenomena. While it is known from observations that some outflows are intrinsically time-dependent (such as protostellar jets), and therefore require some sort of variability to interpret the observations completely, there are yet more fundamental reasons why time-dependent simulations will be crucial. For example, it has recently become clear that the Balbus-Hawley (1991, hereafter BH) instability in accretion disks (which is probably responsible for providing angular momentum transport, and therefore controls the internal dynamics of the disk in most circumstances) can act as a dynamo, amplifying weak initial seed fields up to equipartition with the thermal pressure. Thus, the accretion disk is able to generate its own magnetic field. Understanding how this disk dynamo generated field contributes to an MHD outflow will largely depend on time-dependent simulation.

In this review paper, I will summarize some of the contributions that timedependent simulations have made to our understanding of MHD outflows from disks. I will confine the discussion to multidimensional and time-dependent calculations. Often numerical methods play an important role in the solution of the steady-state problem (e.g. numerical solutions to the Grad-Shafranov equations to compute the multi-dimensional structure of the magnetic field), however, such applications are beyond the scope of this review. 


\section{Numerical Techniques}

Time-dependent, numerical simulations of MHD winds from disks require solution of the equations of MHD, which can be written

$$
\begin{gathered}
\frac{D \rho}{D t}+\rho \nabla \cdot \mathbf{v}=0, \\
\rho \frac{D \mathbf{v}}{D t}=-\nabla p-\rho \nabla \Phi+\frac{1}{4 \pi}(\nabla \times \mathbf{B}) \times \mathbf{B}, \\
\rho \frac{D}{D t}\left(\frac{e}{\rho}\right)=-p \nabla \cdot \mathbf{v}, \\
\frac{\partial \mathbf{B}}{\partial t}=\nabla \times(\mathbf{v} \times \mathbf{B}) .
\end{gathered}
$$

Here, each symbol has its usual meaning ( $e$ is the internal energy density). Note that as written, infinite conductivity is assumed (the ideal MHD limit), so that the magnetic field will be "frozen-in" to the fluid. Nearly all studies of winds from disks presented to date have made this approximation, although both MacLow et al (1995) and Brandenburg et al (1995) considered the effect of ambipolar diffusion on the growth of the $\mathrm{BH}$ instability in the strong coupling limit, and more recently Hawley \& Stone (1996) have performed fully two-fluid simulations of the BH instability in a weakly magnetized disk. One expects nonideal MHD effects to be most important in cold disks, which are very weakly ionized (such as occur in protostellar systems). There remains much work to be done in investigating non-ideal MHD systems.

A wide variety of numerical techniques have been adopted by different investigators to solve equations [1]-[4]. These include Lax-Wendroff, upwind methods combined with the vector potential approach for the field, upwind methods combined with the MOC-CT technique for the field, and first-order Godunov methods. There are important and substantial differences between the numerical diffusivity of these methods, and the way that the divergence-free constraint for the field is enforced. These differences might be important in some applications. However, the underlying physical effects being studied in the disk-jet problem (i.e. rotation, shear, and Lorentz forces) are by and large captured to a similar degree with each of these implementations. Thus, while there are some applications where numerical effects might be important, it is probably safe to say that for the results summarized here, it is not important which particular numerical technique was used.

\section{Results from Simulations}

In order to identify the kinds of problems that can be answered by previous simulations, it is useful to classify them into four categories based on the global nature of the simulation:

1. axisymmetric global models,

2. axisymmetric simulations of the wind only 


\section{3. three-dimensional local models}

\section{4. three-dimensional global models}

Results from each of these four categories of simulations is described below.

\subsection{D Global Models}

The earliest numerical studies of the evolution of magnetized disks adopted the assumption of axisymmetry (which confines the evolution to two spatial dimensions), and utilized large computational domains which encompassed a large radial segment of the disk (to study the global evolution). The first comprehensive study was reported by Uchida \& Shibata (1985, hereafter US) and Shibata \& Uchida (1986, hereafter SU). They used a Lax-Wendroff code to study the timedeperident evolution of a magnetized disk which was initially in sub-Keplerian rotation and embedded in a low density, hot corona in hydrostatic equilibrium. The initial field geometry was assumed to be purely vertical. Initially, the lack of rotational support of the disk caused it to collapse inward on a free-fall timescale. US noticed two effects could cause axial outflows to be produced in their simulations. The first was the radial pinch of the vertical field lines caused by the collapse of the disk could cause coronal plasma to be squeezed out along the axis, much like toothpaste being squeezed from a tube. This effect US termed the "sweeping magnetic pinch". A second effect was the rotational shear between the disk and non-rotating corona generated a strong toroidal field at the disk boundary. This shear, which propagates along the axial field lines as a nonlinear torsional Alfven wave, could also produce outflow via an effect US termed the "sweeping magnetic twist". In fact, both effects occurred simultaneously in the simulations, and SU provide an in-depth analysis of the vertical forces acting on the fluid during their simulations to identify the relative importance of each.

While SU provided a limited number of simulations which examined the time-evolution of disks initially in Keplerian rotation, their primary focus was on sub-Keplerian disks. A similar study, but with a primary focus on Keplerian disks, was presented in Stone \&. Norman (1994). Beginning from nearly identical initial conditions for the disk as US, these studies examined the effect of changing the magnetic field strength on the evolution of the disk. For strong axial fields, the evolution is easy to predict: shear between the disk and corona will generate torsional Alfven waves which should remove angular momentum from the disk on a magnetic braking timescale. For sufficiently strong fields (i.e. $\beta \sim 8 \pi p / B^{2}<$ 1 ), the braking time becomes comparable to the free-fall time; thus the disk should collapse in a few orbital times. Indeed, this is observed in the simulations. The left panel of figure 1 shows the poloidal field lines at $t=2$ orbits in a strong-field simulation. The field lines are pinched in at the equator by the radial collapse of the disk, producing the expected hourglass shape for the field. One might expect for weak fields, i.e. $\beta \sim 8 \pi p / B^{2}>1$, that the magnetic braking timescale would become so long that an initially Keplerian disk will undergo essentially no evolution. Instead, however, radial collapse on a free fall timescale is again observed in the simulations. Stone \& Norman (1994) interpret this collapse as being caused by nonlinear axisymmetric modes of the $\mathrm{BH}$ instability. Perhaps the clearest evidence of this is presented in the right panel of figure 1, which again shows poloidal field lines at $t=2$ orbits but for the 
weak initial field simulation. Note that dramatic evolution of the disk (which is confined to a narrow solid angle at the equator) has occurred. Field lines pinch inwards near the two surfaces of the disk, but in the midplane they bow outwards. MHD simulations by Hawley \& Balbus $(1991 ; 1992)$ have shown that in two-dimensions, the nonlinear outcome of the BH instability is the "channel solution" consisting of two radial streams of fluid, one moving inwards with a deficit of angular momentum compared to Keplerian, and the other moving outwards with an excess of angular momentum. The geometry of the poloidal magnetic field lines clearly show channel solution in the disk, and additional diagnostics of the evolution provided in SN confirm this interpretation. These simulations clearly demonstrate the roles of external (magnetic braking) and internal (BH instability) torques on the evolution of magnetized disks.

More recently, Matsumoto et al (1996) have presented similar studies of thick accretion tori; the prescence of the $\mathrm{BH}$ instability, and its effect on the evolution of the disk, is clearly demonstrated. It is clear that once it develops, the channel solution produces high mass accretion rates in the disk, and thus may have relevance to outburst phenomena. However, Balbus \& Hawley (1992) have demonstrated non-axisymmetric modes of the $\mathrm{BH}$ instability exist, and moreover in three dimensions (3D) the presence of a parasitic instability causes the channel solution to break down into MHD turbulence. These results motivate fully 3D studies of weakly magnetized disks which are BH unstable (see $\S \S 3.3$ and 3.4 below). Most recently, Bell \& Lucek (1995) have studied the global evolution of disks including a softened gravitational potential to examine the effect of thermal pressure gradients produced by radial collapse of the disk.

\subsection{D Wind Region Only}

Because of the complex evolution of axisymmetric MHD simulations of accretion disks (no simulations yet show relaxation to a steady-state), it is difficult to identify and investigate steady-state wind mechanisms, such as the magnetocentrifugal effect discussed by BP. Thus, a number of workers have undertaken time-dependent numerical studies of the wind region only. In these simulations, the disk is treated as a fixed boundary condition at which the magnetic and velocity components, pressure, and density are specified and held fixed. The advantage to such an approach is that the wind can be evolved to a steadystate, and direct comparison to analytic (self-similar) theory can be made. The disadvantage is, of course, that back reaction of the wind on the disk is not accounted for, and moreover, dynamic processes within the disk itself, which may be important for the production of the wind, cannot be studied.

The first results from this approach published in the literature are those of Ustyugova et al (1995). More recently, Ouyed, Pudritz \& Stone (1996) and Romanova (these proceedings) have extended these results. In addition, Dave Meier and his collaborators are completing an extensive study of the parameter space available to outflows (Meier, these proceedings). In every study, the wind is observed to reach a steady state for some region of parameter space. Transitions through each of the three critical points available to an MHD wind are noted in the simulations, and collimation of the flow near or beyond the fast magnetosonic point is observed. Generally such simulations begin with a corona above the disk which is in magnetohydrostatic equilibrium. However, the magnetocentrifugal 
effect operating along field lines inclined at angles of greater than $30^{\circ}$ to the vertical produces strong outflow, and eventually the flow relaxes to a steady state. In some of the models of Ouyed, Pudritz \& Stone, the wind remains episodic for long dynamical times, indicating that no steady-state is possible for the assumed boundary conditions. These episodic solutions may have direct relevance to time-dependent jets, depending on the extent that the conditions at the base of the wind are determined by internal processes in the disk, as opposed to the wind acceleration mechanism itself. Generally good agreement between the predicted location of the Alfven critical point along magnetic field lines from steady-state theory, and that actually observed in the simulations, is found. Typical terminal speed for the flow are at an Alfvenic Mach number of $\sim 5$. It would seem such simulations will be an important tool for studying properties of steady-state winds which are difficult to examine analytically. At the same time, it will be important to merge these simulations with those which treat the internal dynamics of the disk as well, for reasons described in the next section.

\subsection{D Local Models}

As discussed in $§ 3.1$, in 3D parasitic instabilities cause the nonlinear outcome of the BH instability in two dimensions (the channel solution) to break down into MHD turbulence (Goodman \& Xu 1995). In homogeneous boxes (which are equivalent to very small patches internal to the disk) local $3 \mathrm{D}$ simulations of the nonlinear evolution of the BH by Hawley, Gammie, \& Balbus (1995; 1996) have demonstrated the instability results in MHD turbulence with a power spectrum consistent with Kolmogorov. This turbulence is associated with a vigorous angular momentum transport rate, and moreover, the instability can act as a dynamo, amplifying weak seed fields to near equipartition with the thermal energy density for times which are very lng compared to dissipative timescales. Both Brandenburg et al (1995) and Stone et al (1996) have presented 3D simulations of the BH instability in stratified disks. These simulations still represent local patches of a disk in the horizontal plane (i.e. the radial structure of the disk is not included), but incorporate vertical stratification of the disk for several scale heights, making them "quasi-global" in nature. Both groups find that stratification does not change most of the properties of the instability reported for homogeneous boxes (e.g. MHD turbulence still results). In addition, it is found that buoyancy is not an important saturation mechanism; instead local dissipation dominates. However, buoyancy does affect the resulting vertical structure of a BH unstable disk. For example, figure 2 is a "space-time" plot of various quantities during the evolution of a $3 \mathrm{D}$ simulations of a stratified disk with an initially vertical field. The plot is created by averaging each quantity over a horizontal plane, and plotting the result (which is still a function of vertical coordinate $Z$ ) versus time. For times $t<2$ orbits, the linear growth and saturation of the instability as the channel solution is evident. However, the channel solution clearly breaks up at about 3 orbits, and thereafter each variable shows complex structure. However, diagonal streaks which begin at the disk midplane are evident in several variable, especially the magnetic energy. These streaks represent regions of high magnetic field strength which are rising vertically through the disk. Interestingly, while this buoyancy rise of magnetic 
field has no discernible effect on the saturation amplitude of the instability, it does modify the vertical structure of the disk to produce a strongly magnetized corona which surrounds a weakly magnetized core. Figure 3 plots the vertical profile of various quantities deep in the nonlinear regime of the simulations shown in Figure 2. Notice that the plasma- $\beta$ parameter demonstrates this core-corona structure very clearly; near the midplane of the disk $\beta \sim 100$, whereas higher up $\beta<1$. Stone et al (1996) and Brandenburg et al (1995) report a variety of simulations with different vertical boundary conditions in which the same structure is evident, thus it is unlikely the effect is produced by the boundary conditions alone.

What are the implications of these results for the production of MHD winds from disks? The production of a strongly magnetized corona by dynamo action in the disk implies that the field which drives an outflow may be self-generated. Because current simulations have been confined to local patches of the disk, there is still no evidence that large scale, global fields which are sufficiently strong to produce outflows result from the instability. However, because the power spectrum of the turbulence is a decreasing power law, most of the energy in the field is on the largest scales. Because the core of the disk is turbulent, however, random motions of the footpoints may introduce significant Poynting flux, which might affect the acceleration of the flow near the disk. Examining the large scale structure of the field, and whether it still contains significant fluctuating component at high- $Z$ are both vital questions to be addressed by further studies.

\subsection{D Global Models}

Clearly, the most complete numerical models of weakly magnetized disks will be those which can study the global structure and evolution of the disk in $3 \mathrm{D}$. Already, early attempts at such models are underway. Matsumoto (these proceedings) presented a 3D simulation which is an extension of his earlier 2D models of thick tori (Matsumoto et al 1996). The need to achieve sufficient resolution in the disk to capture the MHD turbulence arising from the $\mathrm{BH}$ instability, and yet cover a computational domain large enough so as to address questions regarding the global structure, makes these simulations computationally challenging. There is no doubt, however, that the increase in computing power and increase in sophistication of numerical algorithms will make these studies practical in a very short time.

What will be more difficult is to understand how the evolution of the inner disk depends on the inner boundary condition. In many circumstances accretion occurs onto a strongly magnetized central object. The magnetosphere of this object can truncate the inner disk far from the surface, and the resulting dynamical interaction of the disk and magnetosphere can determine not only the resulting accretion flow, but also the torque on the star. It is not merely a matter of deriving a numerical boundary condition for the inner edge of the disk to allow for realistic global evolution. Instead, the disk-magnetosphere interaction is an important problem in its own right. Moreover, the observation that in many case outflows from accretion disks reach terminal speeds which are comparable to the escape velocity of the central object indicates that at least some fraction of the outflow arises from the disk-magnetosphere interaction region. 
Not only are 3D global models required to study disk winds, but these models must be merged with studies of the disk-magnetosphere interaction region to build a complete picture of outflows from accreting systems. It is encouraging to note that an entire session (session 6 ) at this meeting was devoted to the the disk-magnetosphere interaction region.

\section{Conclusions}

Numerical simulations of MHD winds from accretion disks have begun to make important contributions to our understanding of the outflow phenomena. Perhaps the most important results presented to date have been studies of the nonlinear regime of the $\mathrm{BH}$ instability (see also the review of Hawley, these proceedings), results which are not accessible by any means except numerical methods. The presence of MHD turbulence in the disk, and of dynamo action which may produce a strong disk magnetic field may have important consequences for the wind production mechanism. Fully $3 \mathrm{D}$ global simulations of $\mathrm{BH}$ unstable disks which investigate these effects are certain in the near future.

Acknowledgments. I thank my colleagues Steve Balbus, Charles Gammie, John Hawley, and Mike Norman for their significant contributions to many of the results described here. I thank the meeting organizers for travel support. This work was partially supported by the NSF through grant AST9528299.

\section{References}

Balbus, S.A., \& Hawley, J.F. 1991, ApJ, 376, 214 (BH)

Balbus, S.A., \& Hawley, J.F. 1992, ApJ, 400, 610

Bell, A.R., \& Lucek, S.G. 1995, MNRAS, 277, 1327.

Brandenburg, A., Nordlund, A., Stein, R.F., \& Torkelsson, U. 1995, ApJ, 446, 741.

Blandford, R.D., \& Payne, D.G. 1982, MNRAS, 199, 883.

Eggum, G.E., Coroniti, F.V., \& Katz, J.I. 1988, ApJ, 330, 142.

Fukue, J. 1990, PASJ, 42, 793.

Goodman, J., \& Xu, G. 1994, ApJ, 432, 213.

Hawley, J.F., \& Balbus, S.A. 1991, ApJ376, 223.

Hawley, J.F., \& Balbus, S.A. 1992, ApJ400, 595.

Hawley, J.F., Gammie, C.F., \& Balbus, S.A., 1995, ApJ, 440, 742.

Hawley, J.F., Gammie, C.F., \& Balbus, S.A., 1996, ApJ, 464, 690.

Hawley, J.F., \& Stone. J.M. 1996, in preparation.

Heyvaerts, J., \& Norman, C.A. 1989, ApJ, 347, 435.

Icke, V. 1980, AJ, 85, 329.

Lovelace, R.V.E., Wang, J.C., \& Sulkanen, M.E. 1987, ApJ, 315, 504.

MacLow, M.-M., Norman, M.L., Königl, A., \& Wardle, M. 1995, ApJ, 442, 726.

Matsumoto, R. et al 1996, ApJ, 461, 115.

Ouyed, R., Pudritz, R.E., \& Stone, J.M., 1996, submitted. 
Pelletier, G., \& Pudritz, R.E., 1989, ApJ, 394, 117.

Shibata, K., \& Uchida, Y. 1986, PASJ, 38, 631.

Stone, J.M., \& Norman, M.L. 1994, ApJ, 433, 746.

Stone, J.M., Hawley, J.F., Gammie, C.F., \& Balbus, S.A. 1996, ApJ, 463, 656.

Uchida, Y., \& Shibata, K. 1985, PASJ, 37, 515.

Ustyugova, G.V., et al 1995, ApJ, 439, L39.

Wardle, M., \& Königl, A., 1993, ApJ, 410, 218.

\section{Discussion}

M. Begelman: If the field structure in the corona is very messy, with many small scale field reversals, then the forces among neighbouring flux tubes are likely to far exceed the mean magneto-centrifugal forces. Does this mean that magneto-centrifugal forces are unlikely to be important?

$J$ Stone: It is important to remember that the MHD turbulence driven by the Balbus-Hawley instability is characterised by a power spectrum which is a decreasing power law, so that most of the energy is on the largest scales. As one gets higher in the corona and as the plasma $\beta$ decreases, the instability will be suppressed. Perhaps in this region dissipation on small scales can decrease the tangling in the field and only the larger scale, more ordered component will remain. This field might give rise to a magneto-centrifugal outflow. Clearly, however, we need global simulations that extend higher above the disk than current simulations to test these ideas.

H. Zinnecker: You have not mentioned the works of Camenzind et al. and Shu et al. on MHD jet formation. Could you briefly comment on them?

J. Stone: As I mentioned in the Introduction, my talk today was focused on time-dependent simulations of MHD winds from disks. Both Frank Shu and his collaborators, and Max Camenzind and his collaborators, have constructed steady-state models for MHD winds, a topic I think Arieh Konigl will be reviewing. In general, though, I think it is very significant that steady-state solutions for MHD winds are possible. I think time-dependent simulations will be important to determine whether such solutions are realisable from general initial conditions and whether such solutions are stable. 